\title{
ESTABILIDAD DE LA ESTIMACIÓN DE LA ONDA ANUAL EN ESCALA DIARIA DE LA TEMPERATURA
}

\author{
GUSTAVO NAUMANN, WALTER MARIO VARGAS
}

\author{
Universidad de Buenos Aires, Departamento de Ciencias de la Atmósfera y los Océanos y CONICET, \\ Buenos Aires, Argentina
}

gnaumann@at.fcen.uba.ar, vargas@at.fcen.uba.ar

Recibido Junio de 2010 - Acepto Abril de 2012

\section{RESUMEN}

La elección del método de estimación de efectos determinísticos dentro de series climáticas es el punto de partida de la mayoría de los estudios sobre variabilidad climática. Este análisis es más sensible cuando se desea explorar sobre la presencia de fenómenos no lineales.

Por esto, en este trabajo se analiza la robustez y estabilidad de diferentes métodos para estimar la onda anual en series de temperatura diaria. Se comparan distintos estimadores robustos y se muestra que el cálculo de la onda anual a partir de la mediana produce una estimación más robusta para períodos de información menores a 50 años. Sin embargo, para períodos mayores de información no se observan diferencias entre los distintos estimadores.

Respecto a la persistencia de las series de temperatura se muestra que existe una dependencia entre el tiempo de independencia $\left(T_{o}\right)$ y el período analizado (existe una variación interanual de $T_{o}$ que puede variar entre 3 y 7 días). Además se observa un mayor $T_{o}$ si se considera el término no lineal de las anomalías, es decir el análisis de ésta componente conduce a estimar efectos más persistentes que se ven afectados por la pendiente de la onda anual.

Se muestra la presencia de asimetrías en la onda anual de temperatura que en algunos casos son producto de interacciones no lineales entre la onda anual y las perturbaciones. Por ultimo, los procesos que muestran una mayor dependencia con la onda anual evidencian una mayor persistencia de las anomalías cálidas. Esto esta asociado a procesos advectivos lentos donde las masas de aire cálido permanecen durante varios días, efecto que se evidencia en mejor medida sobre la temperatura mínima. Por esto se propone que en general, la estimación de las anomalías respecto de la onda anual debe contemplar los efectos independientes (lineales) y dependientes (no lineales) de la onda anual. Palabras claves: Temperatura, tiempo de independencia, escala diaria

\footnotetext{
ABSTRACT: ESTIMATION OF THE ANNUAL CYCLE OF TEMPERAURE AND ITS STABILITY AT DAILY TIME SCALE

The choice of estimation of deterministic effect method in climate series is usually the starting point for most studies on climate variability. This decision is more sensitive when the presence of nonlinear phenomena is explored.

Therefore, this paper analyzes the robustness and stability of different methods to estimate the annual cycle in time series of daily temperature. Robust estimators are compared and show that the calculation of the annual cycle using the median is the more robust estimate for reporting periods of less than 50 years. However, for longer periods of information no differences between the various estimators were observed.

Regarding the persistence of the temperature series, there is a dependency between the time of independence $\left(T_{o}\right)$ and the period under review (there is a variation of $T_{o}$ which can vary between 3 and 7 days). In addition, the non-lineal component of the anomalies tend to represent higher values of To if compared with the linear departures, i.e. the analysis of this component leads to estimate long lasting effects that are affected by the slope of the annual cycle.
}

Asymmetries in the annual temperature cycle in some cases are the result of nonlinear interactions 
between the annual wave and disturbances. Finally, the processes that show higher dependence in the annual cycle are characterized by greater persistence of warm anomalies. This is associated with slow advective processes where warm air masses remain for several days. This effect is better represented for minimum temperature. Therefore it is proposed that in general, the estimation or design of the anomalies with respect to the annual wave should be considered as a series expansion that considers the independent effects (linear) and dependent (nonlinear) of the annual cycle.

Keywords: Temperature, Time of independence, Daily time scale

\section{INTRODUCCIÓN}

En los métodos estadísticos relacionados con la construcción de modelos, se asume que las observaciones varían de forma independiente. Sin embargo para muchas variables del sistema climático existe una dependencia entre observaciones y la naturaleza de esta dependencia tiene interés por si misma (Box y Jenkins, 1970).

En términos generales la dependencia puede ser descrita por una función de transferencia $\mathrm{F}(\mathrm{t})$ que se define como la relación $\mathbf{x}_{\mathbf{t}+\mathbf{1}}=\mathbf{F}(\mathbf{t}) \mathbf{x}_{\mathbf{t}}+\varepsilon$ donde $\mathbf{x}_{\mathbf{t}}$ representa el valor de la serie para el tiempo ty $\varepsilon$ el error (Jenkins y Watts, 1968). En general los métodos utilizados para estimar la función de transferencia basada en perturbaciones sinusoidales, han sido siempre exitosos. Esto es porque para perturbaciones de magnitud relevante la respuesta del sistema está enmascarada por perturbaciones incontrolables referidas como ruido (Box y Jenkins, 1970).

Como en la mayoría de las variables climáticas la función de entrada es la onda anual astronómica para una latitud dada y a través de la función de transferencia asociada a éste proceso se obtiene la onda anual de temperatura. En general, los procesos transitorios de alta frecuencia pueden ser considerados como ruido respecto de la onda anual. Sin embargo, existen algunos procesos que por su recurrencia y magnitud afectan la función de transferencia. Estas fluctuaciones de origen estadístico se pueden definir como ruido climático (Leith, 1973; 1978).

La fluctuación anual de la temperatura es una de las oscilaciones periódicas más conocidas en climatología (Köppen, 1948; Trenberth, 1983) siendo ésta una respuesta directa de la radiación solar incidente, Selllers (1965). En particular las series de temperatura están dominadas por éste efecto determinístico (explica entre el 70 y el $90 \%$ de la varianza total de la serie), es decir es un efecto que no varía año tras año (Brooks y Carruthers, 1953; Strauss 1983).

Aunque se reportan tendencias en la fase y amplitud de la onda anual (Thomson 1995; Mann y Park 1996; Spark. y Menzel 2002), las causas y el significado de estos cambios siguen siendo poco conocidos, en parte porque se carece de una comprensión de la variabilidad natural (Stine et al, 2009). Sin embargo, las principales causas de los cambios en la amplitud de la onda anual esta dada por cambios en la dinámica de los efectos transitorios y no por cambios en la onda astronómica.
Por esto es posible considerar un sistema de referencia fijo (determinístico) a partir del cual se pueda estimar la magnitud de las anomalías.

Este estudio se centra en caracterizar el error de estimación de las anomalías de temperatura diaria con el objetivo de disminuir los errores asociados al pronóstico objetivo de las perturbaciones de estas series. Por esto, en este trabajo se explora sobre la robustez y estabilidad de las estimaciones de la onda anual y de las funciones de transferencia de las series de información diaria de temperatura máxima (Tx) y mínima (Tn) en San Miguel de Tucumán (1891-2005).

Además se analiza el ciclo anual conjunto entre la temperatura máxima y mínima a partir de la representación vectorial de las funciones de Fourier asociadas a las series. Esto se realiza para sintetizar el ciclo anual en sus aspectos más importantes, los extremos diarios de temperatura. Por otra parte se trata de obtener conclusiones sobre distintas formas de estimación del ciclo y analizar las peculiaridades del mismo dadas por las tasas de cambio de dicha función.

El trabajo se organiza de la siguiente manera: Los datos utilizados en este análisis se describen en la sección 2. En la sección 3 se propone una descripción sobre los métodos de descomposición de series temporales y se discute sobre la estabilidad temporal de las estimaciones de los métodos de filtros de la onda anual. La estabilidad y robustez de las estimaciones de función de autocorrelación y tiempo de independencia se discute en la sección 4 . Finalmente en la sección 5 se resumen las principales conclusiones.

\section{DATOS Y MÉTODOS}

Para efectuar un análisis consistente de sensibilidad en la estimación de la onda anual es necesario disponer de series temporales homogéneas que cuenten con información diaria en lo posible durante el período instrumental. Para este estudio se ha seleccionado la serie de referencia de acuerdo a la longitud de su registro, calidad de medición y control de calidad y niveles de consistencia conocidos (Vargas y Naumann, 2008). Así mismo, es posible encontrar una descripción sobre las tendencias y persistencia sobre estas series en Vargas et al. (2006) y Naumann et al. (2011). 
Las series de temperatura máxima y mínima de San Miguel de Tucumán se han seleccionado para el presente análisis. Esta estación dispone de más de un siglo de información diaria (En la tabla 1 se muestra la información asociada a esta localidad).

La descomposición de series temporales permite identificar importantes propiedades de la información contenida en éstas a través un diagnostico objetivo. Como primer aproximación a la descomposición se asume que las series de tiempo pueden ser descompuestas en tres componentes básicas:

tendencias

estacionalidad

las variaciones irregulares o efectos transitorios que permanecen en las series una vez removidos la tendencia y el efecto estacional.

En general se desea definir un sistema de referencia a partir del cual efectuar el cálculo de las anomalías. Surge esto como prioridad inicial del análisis ya que la elección defectuosa del filtro puede inducir a la generación de ruido o periodicidades espurias.

La estacionalidad es la porción de la variación debida a factores que recurren sistemáticamente una o más veces al año. Un efecto estacional es razonablemente estable con respecto al tiempo en dirección y magnitud. La onda anual astronómica rige principalmente la estacionalidad de la temperatura, siendo factores como la latitud y continentalidad los que definen su amplitud y fase.

A partir de esta característica determinística se desea analizar los apartamientos de las condiciones "normales" que pueden estar producidos tanto por factores internos como externos al sistema climático.

Estos efectos irregulares son evidencia de las fluctuaciones de corto plazo en las series que no son sistemáticas (aunque no necesariamente debido a factores aleatorios) y contienen baja predictabilidad. Sin embargo, los procesos asociados a estos fenómenos transitorios contienen gran cantidad de información y pueden ser utilizados para mejorar la calidad de las predicciones (Miller y Williams, 2003).

Conociendo estos elementos, es posible definir un modelo de representación que muestre una componente estacional más estable, lo cual es lo más apropiado para ajustar a las series. Entre los dos modelos de representación de anomalías utilizados usualmente se encuentra el aditivo y multiplicativo (Chatfield, 1989).

Tabla 1 - Descripción, ubicación geográfica y período de observaciones para la estacione analizada.

\begin{tabular}{ccccc}
\hline Estación & Longitud & Latitud & Inicio & Fin \\
\hline S. M. Tucumán & -65.20 & -26.80 & 1891 & 2007 \\
\hline
\end{tabular}

El modelo de descomposición aditivo asume que las componentes de las series se comportan de manera independiente. Este modelo se utiliza si los efectos estacionales e irregulares son independientes, es decir el efecto de la onda anual no varía con el tiempo.

Matemáticamente, la serie original $\mathrm{x}_{\mathrm{t}}$, en el tiempo $\mathrm{t}$, se considera como la suma de las tres componentes: la tendencia $\mathrm{T}_{\mathrm{t}}$, la estacional $\mathrm{S}_{\mathrm{t}}$, y la irregular $\mathrm{I}_{\mathrm{t}}$.

$$
x_{t}=T_{t}+S_{t}+I_{t}
$$

En el modelo aditivo, cada componente tiene las mismas dimensiones que la serie original. La serie ajustada por estacionalidad se puede expresar como

$$
x_{t}^{\prime}=x_{t}-S_{t}=T_{t}+I_{t}
$$

En éste modelo las variaciones irregulares no son independientes de la onda anual, es decir la magnitud de las anomalías depende directamente de la época del año. La serie $\mathrm{x}_{\mathrm{t}}$, en el tiempo $\mathrm{t}$, es considerada como el producto de las componentes estacional $\mathrm{S}_{t}$, la tendencia $\mathrm{T}_{t}$ e irregular $\mathrm{I}_{t}$

$$
x_{t}=T_{t} \times S_{t} \times I_{t}
$$

En el modelo multiplicativo la tendencia tiene las mismas dimensiones que la serie original, mientras que las componentes estacional e irregular son factores adimensionales centrados alrededor de uno

$$
x_{t}^{\prime}=x_{t} / S_{t}=T_{t} \times I_{t}
$$

Los modelos para determinar cada una de las componentes, generalmente utilizan simultáneamente los filtros de Kálmán o técnicas relacionadas. En Harvey (1990) se encuentra un detallado análisis de estas metodologías. En líneas generales la metodología asigna a cada componente un modelo propio determinado por sus características espectrales. El "Classical Seasonal Decomposition" (CSD) es uno de los métodos más utilizados para descomponer series temporales. Esta técnica es descripta y discutida en detalle en Makridakis y Wheelwright (1989) y Makridakis et al., (1998).

En este trabajo se calcularon las descomposiciones de las series de temperatura mínima en San Miguel De Tucumán según los modelos aditivo y multiplicativo utilizando el método CSD. Si bien se muestra el análisis para el período 1998-2005, los valores estacionales fueron estimados a partir del período completo que se dispone de información diaria (1891-2005), no considerar toda la información disponible o si ésta es escasa puede inducir a errores en la estimación de la estacionalidad debido a que efectos transitorios pueden ser considerados como parte de lo onda anual y por consiguiente su dependencia con ésta será mayor. Este tópico se discutirá en la sección 4, en especial la influencia del período en la estabilidad de la estimación de la onda anual. 


\section{RESULTADOS Y DISCUSIÓN}

En las Figuras 1 y 2 se muestran las componentes $S_{t}$, $T_{t}$ e $I_{t}$ según los dos métodos. Si bien las dos estimaciones muestran el mismo comportamiento de la onda estacional, se observan diferencias significativas en la componente irregular de la serie. Los residuos para el método aditivo no muestran una estacionalidad definida y su magnitud es similar durante todo el año. Esto evidencia que la mayor parte de los efectos que dominan las series son lineales.

Para el método multiplicativo se observa una mayor amplitud de las irregularidades en el invierno, principalmente asociado a irrupciones frías. Así mismo, la menor amplitud de los residuos, principalmente en las estaciones de transición evidencia una mayor dependencia del término no lineal en estas épocas del año. Esto concuerda con lo encontrado en Minetti y Vargas (1997) donde muestran la existencia de una significativa interrelación entre la onda estacional y las perturbaciones debido a una lenta interacción entre las condiciones de borde y la atmósfera.

Por otro lado, para poder caracterizar la onda anual a partir de los promedios diarios es necesario definir parámetros que sinteticen el comportamiento de ésta oscilación. El parámetro más común que define las características térmicas de un día en particular es el valor medio de éste, calculado como la media aritmética. Sin embargo, para obtener una buena estimación de éste parámetro, es necesario que ésta sea estable, para lo cual se introduce la estimación robusta de los modelos de posición.

¿Por que analizar la eficiencia de un estimador? Para solventar las dificultades asociadas a la inestabilidad que presentan los estimadores, se plantea el uso de estimadores dentro del campo de la Estadística Robusta. Esta rama de la
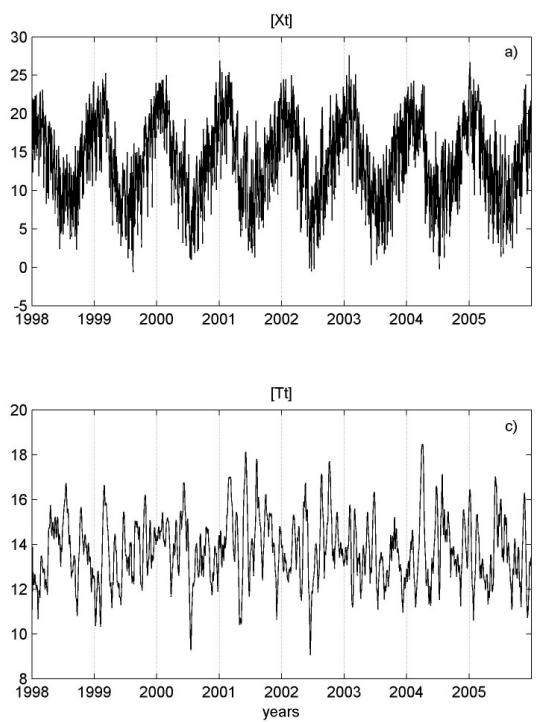

Estadística se centra en el desarrollo y análisis de estimadores que son estables (robustos) frente a cambios en las distribuciones supuestas. Por esto si la variable analizada presenta cambios que pueden estar debidos tanto a efectos naturales como antropogénicos que pueden afectar su distribución, los efectos de ésta inestabilidad se pueden reflejar en la estimación de otros parámetros derivados de estos momentos.

Supongamos sea $\mathrm{F}=\phi$, la función de distribución $\mathrm{N}(0,1)$, entonces las $\mathrm{x}_{\mathrm{i}}$ tienen distribución $\mathrm{N}\left(\mu, \sigma^{2}\right)$. Por lo tanto, un estimador óptimo de $\mu$ es (Hoel, 1984)

$$
\bar{x}=\sum_{i}^{n} \frac{x_{i}}{n}
$$

Sin embargo, en la mayoría de las aplicaciones prácticas a lo sumo se puede asegurar que los errores de medición tienen distribución aproximadamente normal. Por lo tanto cabe preguntarse cual será el comportamiento del estimador $\overline{\mathrm{x}}$ en casos de no-normalidad.

Una de las formas más simples de estimar el parámetro de posición es hacer la media aritmética menos sensitiva a los "outliers" o extremos ajustando o eliminando una porción de datos de cada cola de la distribución y computar la media de los valores centrales remanentes. Esta media ajustada pertenece a una familia de estimadores que puede ser indexada por el parámetro $\alpha$. Este parámetro representa la fracción de observaciones eliminadas en cada cola de la distribución de la muestra. Formalmente, si $x_{1} \leq x_{2} \leq \ldots \leq x_{n}$, es la serie ordenada de una muestra de datos aleatoria, entonces la media $\alpha$-ajustada tiene la forma;

$$
\bar{x}_{\alpha}=\frac{1}{n(1-2 \alpha)}\left\{\sum_{i=k+2}^{n-k-1} x_{i}+(1+k-n \alpha)\left[x_{k+1}+x_{n-k}\right]\right\}
$$
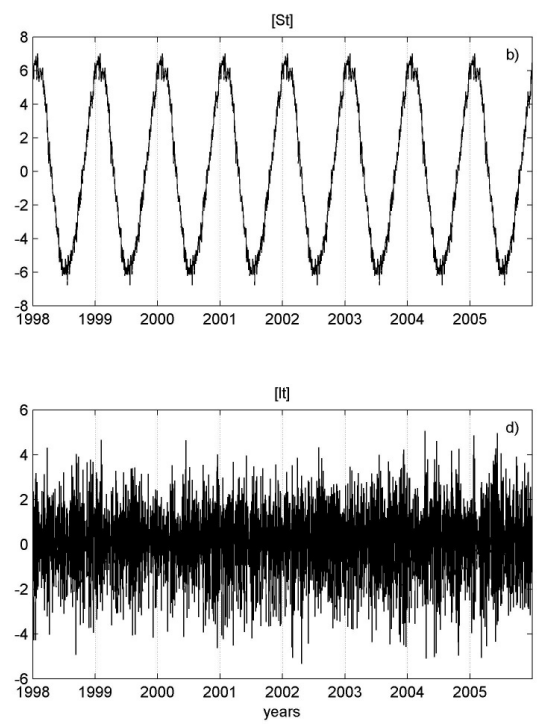

Figura 1 - Descomposición aditiva para la temperatura mínima diaria en OCBA (1998-2005), basado en el período 1910-2005 
si $k+2 \leq n-k-1$. Si ocurre lo contrario,

$$
\bar{x}=\frac{1}{2}\left[x_{k+1}+x_{n-k}\right]
$$

Donde $\mathrm{k}$ es el mayor entero menor o igual que n $\alpha \mathrm{y}$ $0<\alpha<0.5$.

Las medias ajustadas incluyen casos especiales como la media aritmética $(\alpha=0)$, la "midmean" $(\alpha=0.25)$ y la mediana $(\alpha=0.5)$. La elección de $\alpha$ depende de cada distribución, donde la no-normalidad de los datos y el nivel de protección que se desea sobre los "outliers" o extremos juegan el rol más importante. Los valores más frecuentes utilizados para $\alpha$ incluyen a 0 , $0.05,0.1,0.25$ y 0.5 .

Además es de suponer que la estimación de los promedios varíe con la cantidad de datos o años con que se calculan estos, con lo cual la elección de la cantidad de información es un elemento importante para tener en cuenta como filtro de los efectos transientes. Con esto, es posible aplicar ésta estimación (suponiendo $\alpha=0,0.1,0.25$ y 0.5 ) para cada día del año sobre las series de temperatura sobre períodos que varían entre $5 \mathrm{y}$ 120 años. Como primera aproximación se muestran en la Figura 3-a los valores medios anuales de la temperatura mínima del 1 de enero estimados según las Ecuaciones (5) y (6).

Se observa que la estimación del valor medio comienza a estabilizarse a partir de 30-40 años de información. Se observan grandes diferencias entre las primeras estimaciones (períodos de 5 y 10 años) y las estimaciones con 30-40 años de datos, con una magnitud de casi $2{ }^{\circ} \mathrm{C}$. Con esto se podría sugerir que una estimación de la onda anual con 40 años de datos es una buena aproximación a la onda anual astronómica. Sin embargo a partir de 80 años de datos se observa otra región de convergencia de las estimaciones con diferencias entre si de menos de $0.5^{\circ} \mathrm{C}$.
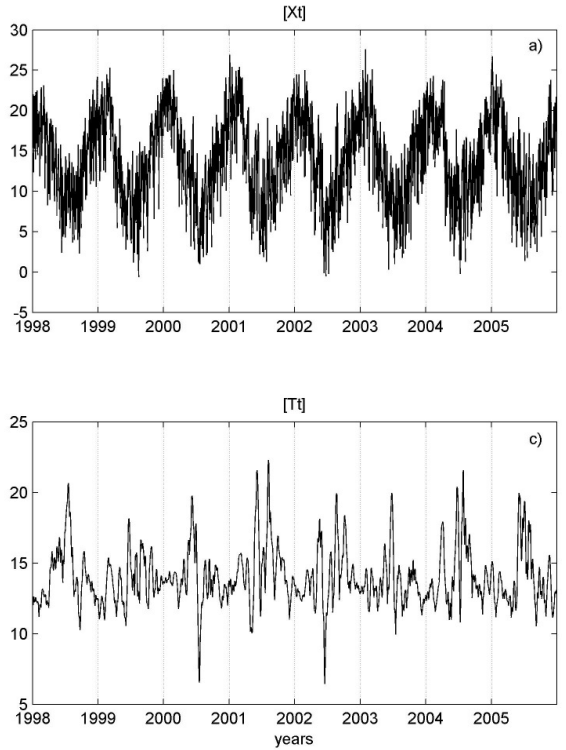

En cuanto a las estimaciones sesgadas con $\alpha=0.25$ y $\alpha=0.5$ parecen converger más rápidamente (aproximadamente 20-30 años) a los valores estimados con mayor cantidad de datos, mientras que los estimadores con $\alpha=0$ y $\alpha=0.1$ muestran menor variación con 40-50 años de información.

Para generalizar los resultados se propone analizar la dispersión que se comete al estimar la onda anual con $\mathrm{N}$-años comparando con la mejor estimación posible con el máximo $\mathrm{N}$ o sea con el período completo de información disponible. En la Figura 3-b se muestran los desvíos de los residuos de la estimación de la onda anual para la temperatura mínima tomando como periodo de referencia el total de años. Las estimaciones de la onda anual para todos los $\alpha$ se comportan de manera similar, aunque la estimación con la mediana es la que converge más rápidamente a la mejor estimación. A partir de esta información es posible determinar el nivel de sensibilidad de la estimación en función de la longitud del período. Por ejemplo, si se tolera un error de $0.5^{\circ} \mathrm{C}$ se requiere un período de 30 años para la mediana y de 40 años para los otros estimadores. Si la sensibilidad requerida es de $0.25^{\circ} \mathrm{C}$ se necesitaran 60 años si se estima a partir de la mediana, mientras que para los otros estimadores se requieren como mínimo 70 años de información diaria. En general para la temperatura se observa que los errores se distribuyen uniformemente tanto a valores positivos como negativos, alcanzando valores entre 3-4 ${ }^{\circ} \mathrm{C}$ en los períodos más cortos.

Así mismo, es posible evaluar la mejora en la estimación de la onda anual en función del incremento en el período de datos a partir del cálculo de la varianza explicada por la onda anual respecto de la variación total. En la Figura 3-c se muestra la varianza explicada por el primer armónico sobre la temperatura mínima. Dado el alto grado determinístico de
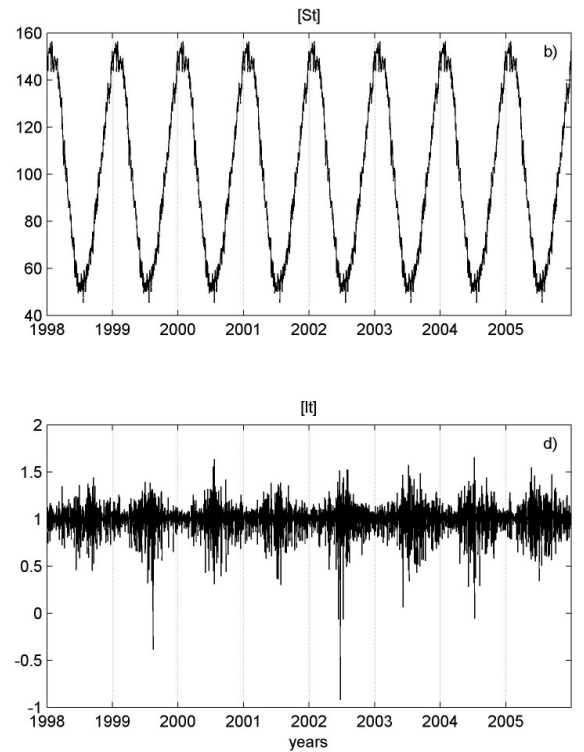

Figura 2 - Descomposición multiplicativa para la temperatura mínima diaria en OCBA (1998-2005), basado en el período $1910-2005$. 
la onda astronómica se observa que para el período de menor longitud (cinco años), ésta ya se explica cerca del $90 \%$, a pesar de la presencia de efectos transitorios. A pesar de esto se observa un crecimiento de la varianza explicada por la onda anual, estabilizándose en valores cercanos al $98 \%$ con períodos de 50-60 años.

Si analizamos la Figura 3-c se tiene que si se estima la onda anual a partir de los promedios diarios, se tiene una mejora en la estimación a medida que se agranda la muestra con que calcula cada promedio diario, llegando hasta valores de varianza explicada del 98\%. El hecho de que la varianza explicada tienda a 98\% cuando la cantidad de información se incrementa se debe principalmente a la presencia de asimetrías en la onda anual observada. Esto se observa dada la presencia de efectos no lineales producidos por fenómenos transientes recurrentes aunque no periódicos que influyen sobre la pendiente de la onda anual a la entrada y salida de las estaciones de transición en la región (Minetti y Vargas, 1997).

La utilización de series de Fourier permite calcular analíticamente una expresión matemática de variables periódicas. Sin embargo es usualmente difícil visualizar interacciones entre variables relacionadas, más allá del estudio de sus gráficos o a través del análisis de los coeficientes. El análisis vectorial es útil para mostrar proyecciones en planos definidos para indicar interacciones entre variables periódicas con el tiempo (Russell, 1984). Si las variables son medidas como cambios por unidad de tiempo y expresadas como series de Fourier, es posible integrar y diferenciar estas expresiones y ser representadas como funciones vectoriales.
Si consideramos dos variables periódicas, se puede expresar en términos de la función vectorial $\mathrm{f}(\mathrm{t})$ y series de Fourier como sigue;

$$
f(t)=\left(\begin{array}{l}
x \\
y
\end{array}\right)=\left(\begin{array}{c}
a_{0}+\sum_{n=1}^{m}\left(a_{n} \cos n t+b_{n} \operatorname{sen} n t\right. \\
c_{0}+\sum_{n=1}^{m} c_{n} \cos n t+d_{n} \operatorname{sen} n t
\end{array}\right)
$$

Si las variables x e y son medidas en términos de cambios por unidad de tiempo, es posible examinar las relaciones entre éstas variables y sus formas diferenciadas como funciones vectoriales. Las series de Fourier se pueden diferenciar (solo si son continuas) termino a termino y la función vectorial es de la forma;

$$
f(t)=\left(\begin{array}{l}
x \\
y
\end{array}\right)=\left(\begin{array}{l}
\sum_{n=1}^{m}\left(-n a_{n} \operatorname{sen} n t+n b_{n} \cos n t\right. \\
\sum_{n=1}^{m}-n c_{n} \operatorname{sen} n t+n d_{n} \cos n t
\end{array}\right)
$$

Donde $\mathrm{f}$ es función del tiempo $\mathrm{t}, \mathrm{x}$ e y son las variables que pueden ser expresadas en términos de series de Fourier; y los coeficientes $a_{n}, b_{n}, c_{n} y d_{n}(n=0, \ldots, m)$ definen la serie de Fourier.

Por ejemplo, para funciones especificas en el espacio tridimensional bajo condiciones especificas donde todos los coeficientes $a_{n}, b_{n}, c_{n}$ y $d_{n}$ son cero, excepto $b_{1}=1$ y $c_{1}=1$, es $\operatorname{decir} \mathrm{x}(\mathrm{t})=\operatorname{sen}(\mathrm{t})$ e $\mathrm{y}(\mathrm{t})=\cos (\mathrm{t})$, y para $\mathrm{b}_{1}=1$ y d $\mathrm{d}_{1}=1 ; \mathrm{x}(\mathrm{t})=\operatorname{sen}(\mathrm{t})$ e $y(t)=\operatorname{sen}(t)$. Para el primer caso el grafico de $f$ es una espiral en torno al eje t. La proyección sobre el eje xy es un circulo de radio 1 , es decir para series periódicas desfasadas en $\pi / 2$ se tiene un proyección circular, mientras que a medida que este
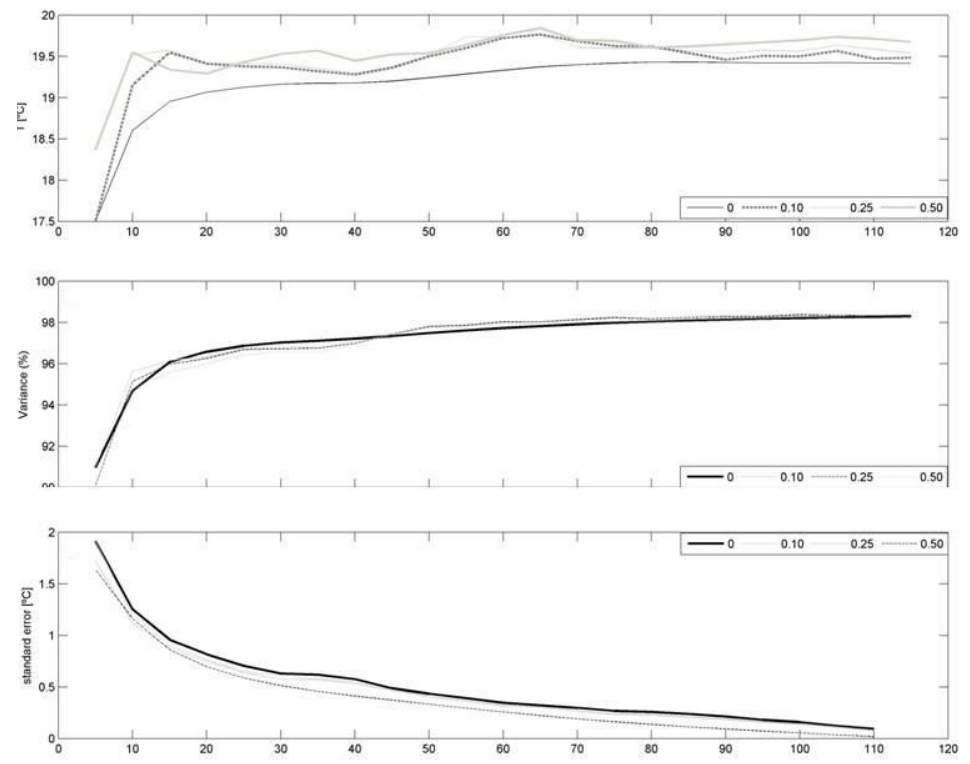

Figura 3 - (a) Estimación de la temperatura mínima del primero de enero en OCBA para períodos entre 5 y 120 años, según las medias ajustadas por $\alpha=0, \alpha=0.10, \alpha=0.25$ y $\alpha=0.5$, (b) desvíos de los residuos de la onda anual estimada de la temperatura mínima con $\mathrm{N}$ años respecto de la onda anual y (c) varianza explicada por el primer armónico de la onda anual de temperatura mínima para estimaciones con distintas longitudes de registros. 
desfasaje cambia, se obtienen proyecciones ovaladas, hasta llegar al segundo caso analizado, donde la proyección sobre el plano $\mathbf{x y}$ es una segmento de pendiente -1 .

La Ecuación 8 se ha utilizado para calcular la función vectorial, cuyas componentes están representadas por los promedios diarios de temperatura máxima y mínima diaria en S. M. de Tucumán. La proporción de la varianza explicada por las series de Fourier para ambas series es superior al $96 \%$. En la Figura 4-a se muestra la proyección de las funciones vectoriales en el plano, asimismo la proyección de los valores de la onda anual calculada con los promedios diarios. La flecha indica la dirección de rotación a través del año. En particular, estas proyecciones caracterizan el régimen climático de las interacciones de Tx-Tn al que pertenece la localidad de Tucumán.

Las mayores diferencias entre las trayectorias se ubican en el invierno donde las estimaciones a partir de las series de Fourier no representan las variaciones de la onda anual al inicio y fin del invierno y al inicio del verano. Este efecto es más claro si se analizan las diferencias entre la estimación a partir de los promedios diarios respecto de las series de Fourier considerando el primer armónico y el primer y segundo (Figura 4-b). Se observa la remanencia de una onda semianual, atenuada parcialmente al considerar los dos primeros armónicos, con las mayores diferencias en las estaciones de transición.

Siguiendo la Ecuación 9 es posible diferenciar las series de Fourier para obtener la tasa de cambio conjunta entre la temperatura máxima y mínima. En la Figura 5 se muestran las proyecciones de las formas diferenciadas para las estimaciones de la onda anual considerando solo el primer armónico y considerando los dos primeros armónicos. Aquí se observa que la tasa de cambio es mayor durante el verano para la estimación con dos armónicos mientras esta tasa es menor durante las estaciones de transición y el invierno. Cabe considerar que errores de estimación en estos cambios puede conducir a errores en la estimación de los máximos (día más calido de la onda) y mínimos (día más frío), es decir en la fase de la onda. En la tabla 2 se muestran las fechas de ocurrencia del mínimo de la onda calculadas como el día de invierno en que la ecuación 9 toma el valor cero. Aquí se observan diferencias entre estimaciones de hasta 7 días principalmente sobre la temperatura mínima. Así mismo se tiene un desfasaje entre las ondas anuales de la temperatura máxima y mínima de aproximadamente 20 días, efecto que da la forma elíptica de la proyección de la función vectorial estimada según la ecuación 8 .

En la Figura 6 se muestra la relación entre las estimaciones de la onda anual de temperatura mínima a partir de los promedios diarios (OA) y las estimaciones a partir del primer armónico (AA), los primeros dos armónico (AA2), el método CSD aditivo y CSD multiplicativo. Se observa que en general todas las estimaciones considerando efectos lineales ajustan bien, las que tienen menor dispersión son aquellas relacionadas con el método CSD y la estimación con los primeros dos armónicos. Pero si se analiza la relación entre la estimación OA y la CSD multiplicativa se observan grandes diferencias principalmente en la representación de los valores extremos.

Dada la presencia de estas asimetrías de la onda anual de temperatura, no se observa una mejora significativa al aplicar los filtros producto del análisis de Fourier, considerando uno o más armónicos. Así mismo estos métodos no son lo suficientemente sensibles para detectar deformaciones importantes en la onda anual, así como singularidades intraestacionales, (Minetti, 1991).

\section{ESTABILIDADY ROBUSTEZ DE LADE FUNCIÓN DE AUTOCORRELACIÓN Y ESTIMACIONES ESPECTRALES}

Las distintas interacciones que existen en las componentes del sistema climático generan perturbaciones y cambios en los
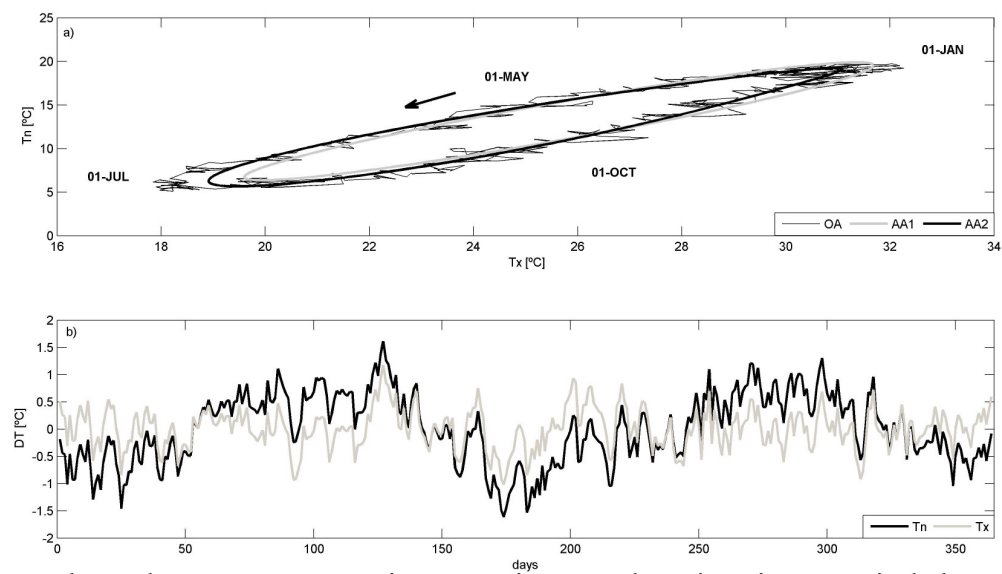

Figura 4 - Proyecciones de la marcha anual para la temperatura máxima y mínima para la estimación a partir de los promedios diarios (negro), el primer armónico (gris) y la suma del primer y segundo armónico (negro grueso) (a) y las diferencias entre la onda anual de los promedios de la Tn y las estimaciones de series Fourier (b). 


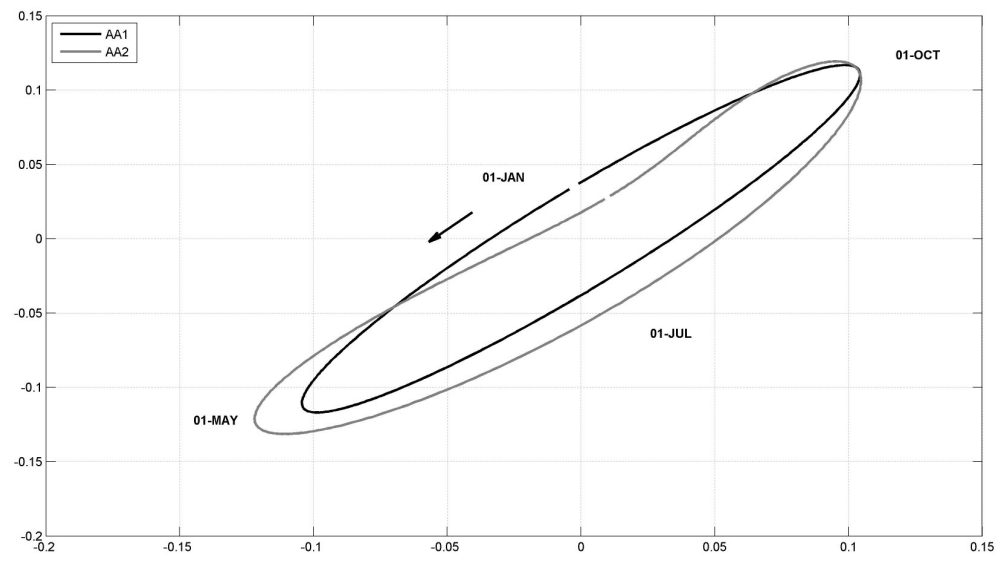

Figura 5 - Proyección de la forma diferenciada de la función vectorial que representa la onda anual considerando el primer armónico (negro) y el primer y segundo armónico (gris).

Tabla 2 - Fecha de ocurrencia del mínimo anual de la onda anual estimada a partir de los promedios diarios (OA), del primer armónico (AA) y de los primeros dos armónicos (AA2).

\begin{tabular}{ccc}
\hline & Tx & Tn \\
\hline OA & 17-Jul & 03-Jul \\
\hline AA & 19-Jul & 30-Jun \\
\hline AA2 & 17-Jul & 26-Jun \\
\hline
\end{tabular}

procesos que están definidos por su persistencia y propiedades espectrales. En particular la proporción entre la relación entre el ruido/señal climáticas esta altamente relacionada con la persistencia en la atmósfera (Leith, 1973, 1978). La función de autocorrelación es una buena herramienta para medir el grado de persistencia o dependencia de las observaciones Trenberth (1984a, b).

Pero, en este caso ¿Existen dependencias entre la función de autocorrelación y el método de filtrado de la onda anual? En la Figura 7 se muestran las funciones de autocorrelación para los años 1998, 2000, 2002 y 2003 de las anomalías de Tn calculadas según los filtros analizados en la sección anterior. Para los filtros aditivos no se observan diferencias significativas entre las estimaciones, con lo cual los procesos físicos caracterizados por la persistencia y memorias remotas no son alterados por el filtro. El autocorrelograma que muestra mayores diferencias es el asociado a las anomalías según el método multiplicativo, aunque si bien en general muestra un comportamiento comparable en cuanto a la detección de picos significativos con respecto a las estimaciones aditivas, las mayores diferencias se observan para el año 1998, donde existe una mayor persistencia. Este resultado indica que en ciertas condiciones las interacciones no lineales entre la onda anual y los efectos transientes pueden producir periodicidades no explicadas por los efectos lineales.

Como primer aproximación parece razonable considerar una serie con la mayor cantidad de información para representar los procesos de alta frecuencia asociados a la persistencia. Pero,
$¿ Q u e$ cantidad de datos es necesaria para obtener una estimación estable de la persistencia? En la Figura 8-a se muestran las diferencias entre el coeficiente de autocorrelación $\left(\mathrm{r}_{\mathrm{k}}\right)$ calculado $\mathrm{y}$ el asociado a un proceso Markoviano $\left(\mathrm{r}_{1 \mathrm{k}}\right)$, con $\mathrm{k}=2, \ldots, 7$, para estimaciones con períodos de distinta longitud. Se evaluó la significancia de la relación a partir de la hipótesis nula que considera $r_{k}-r_{1 k}=0$ con $5 \%$ de confidencia. Se observa que para períodos menores a 50 años, los valores de $r_{k}$ estimados difieren de los que definen el proceso estocástico para todo k. Si se consideran períodos mayores a 50 años se observa que para las autocorrelaciones con lags de 2 a 5 convergen significativamente a los valores teóricos. Para desfasajes mayores a 5 días los valores no convergen a los valores de un proceso markoviano indicando en cierta medida el alcance de la persistencia, es decir el tiempo de independencia entre mediciones.

En particular el conocimiento del tiempo de independencia $\left(\mathrm{T}_{\mathrm{o}}\right)$ entre observaciones es de particular interés en el tratamiento de series temporales asociadas a procesos con persistencia. Bayley y Hammersley (1946) y Trenberth (1984a) sugieren como el numero efectivo de observaciones independientes $\left(\mathrm{N}_{\mathrm{eff}}\right)$ a

$$
N_{\text {eff }}=N \Delta T / T o
$$

Donde $\mathrm{N}$ es el número de datos, $\Delta \mathrm{T}$ es el paso de tiempo y $\mathrm{T}_{\mathrm{o}}$ es el tiempo de independencia

$$
T o=1+2 \sum_{k=1}^{N}(1-k / N) r_{k}
$$

Donde $\mathrm{r}_{\mathrm{k}}$ es la autocorrelación para el lag k. Si se tiene gran cantidad de información, es decir $\mathrm{N}$ es grande se puede aproximar la ecuación (11) a

$$
\text { To } \approx \frac{1+r_{1}}{1-r_{1}}
$$

Resultados similares a lo discutido en la Figura 8-a se obtienen al analizar la estabilidad de la estimación de $T_{o}$ 

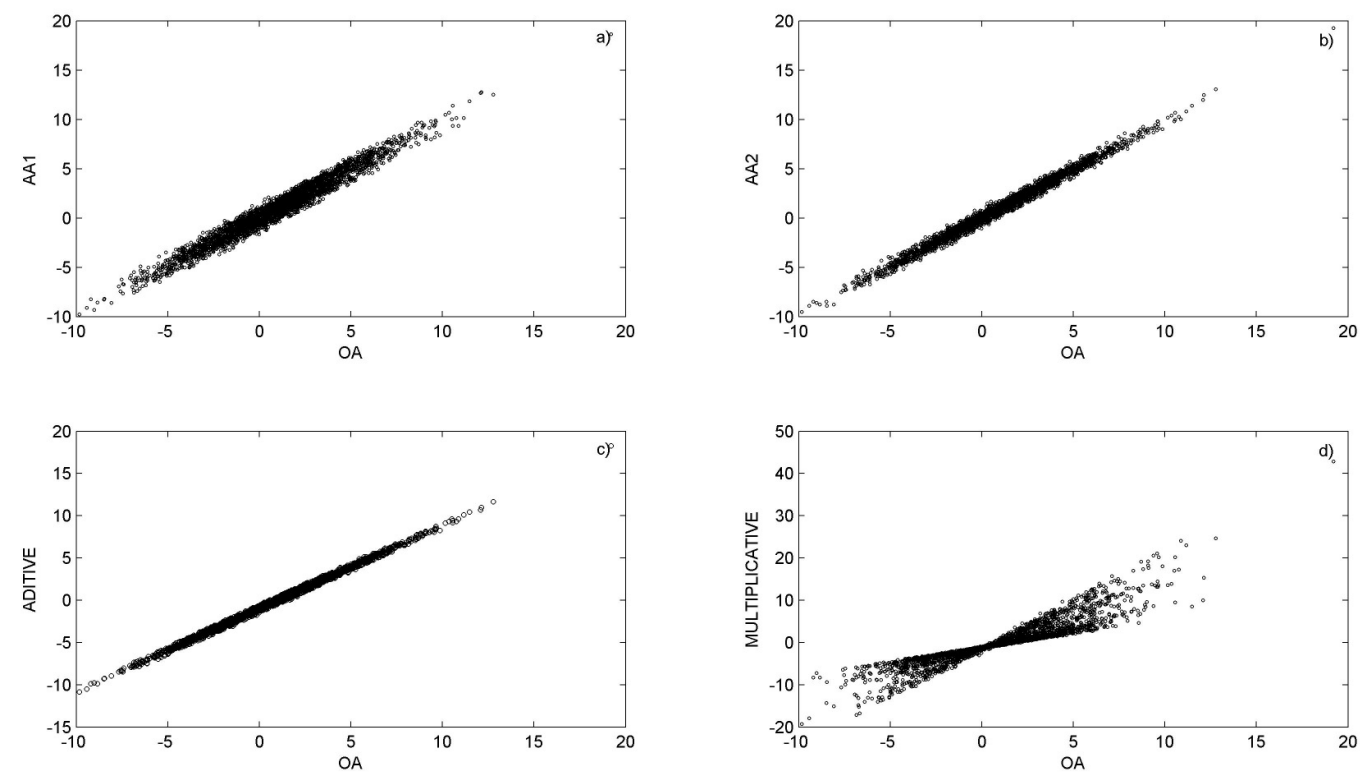

Figura 6 - Relación entre las estimaciones de la onda anual de temperatura mínima en S.M. de Tucumán a partir de los promedios diarios (OA) y las estimaciones a partir del (a) primer armónico (AA), (b) los primeros dos armónico (AA2), (c) el método CSD aditivo y (d) CSD multiplicativo.
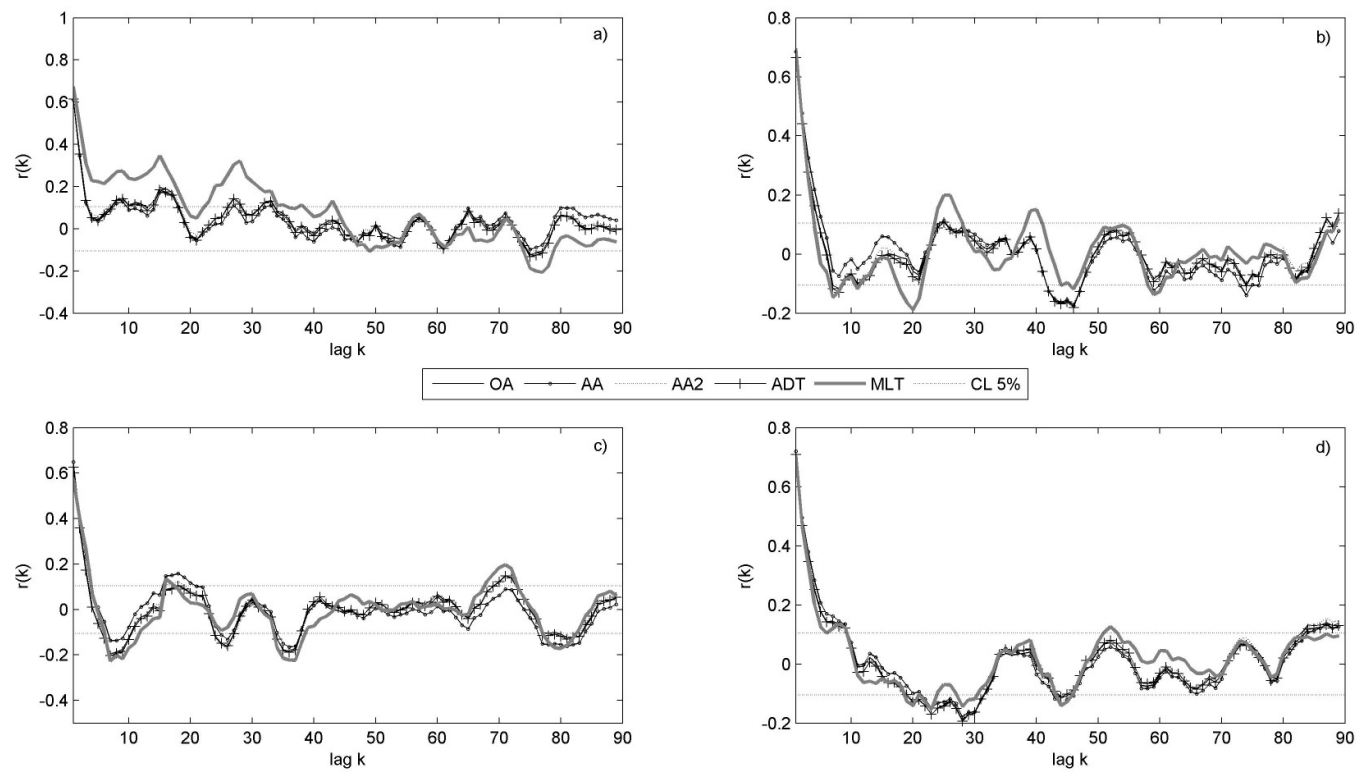

Figura 7 - Función de autocorrelación para las series de anomalías de temperatura mínima en S. M. de Tucumán para los años (a) 1998, (b) 2000, (c) 2002 y (2004) en referencias a las estimaciones de la onda anual según los promedios diarios (OA), el primer armónico (AA), los primeros dos armónicos (AA2) y la descomposición CSD aditiva (ADT) y multiplicativa (MLT).

(Figura 8-b). Se tiene que para periodos mayores a 50 años el valor de $T_{o}$ para la temperatura mínima se estabiliza en 4.8 días para la estimación lineal de anomalías, mientras que para la estimación multiplicativa el valor tiende a ser de 5.6 días. Este resultado implica que existe una dependencia del $T_{o}$ con el período analizado, es decir existe una variación interanual de $T_{o}$ que puede variar entre 3 y 7 días. Minetti (1991) encontró variaciones interanuales del tiempo de independencia de la temperatura máxima en S. M. de Tucumán posiblemente asociados a la alternancia entre períodos húmedo/secos y el efecto que esto tiene sobre la temperatura.

Si se considera el termino no lineal de las anomalías se observa un mayor $T_{o}$, es decir el análisis de ésta componente conduce a estimar efectos más persistentes que se ven afectados por la pendiente de la onda anual. Para los casos mostrados en la Figura 7, el año 1998 es el que muestra mayores diferencias entre las funciones de autocorrelación para las componentes lineal y no lineal. 

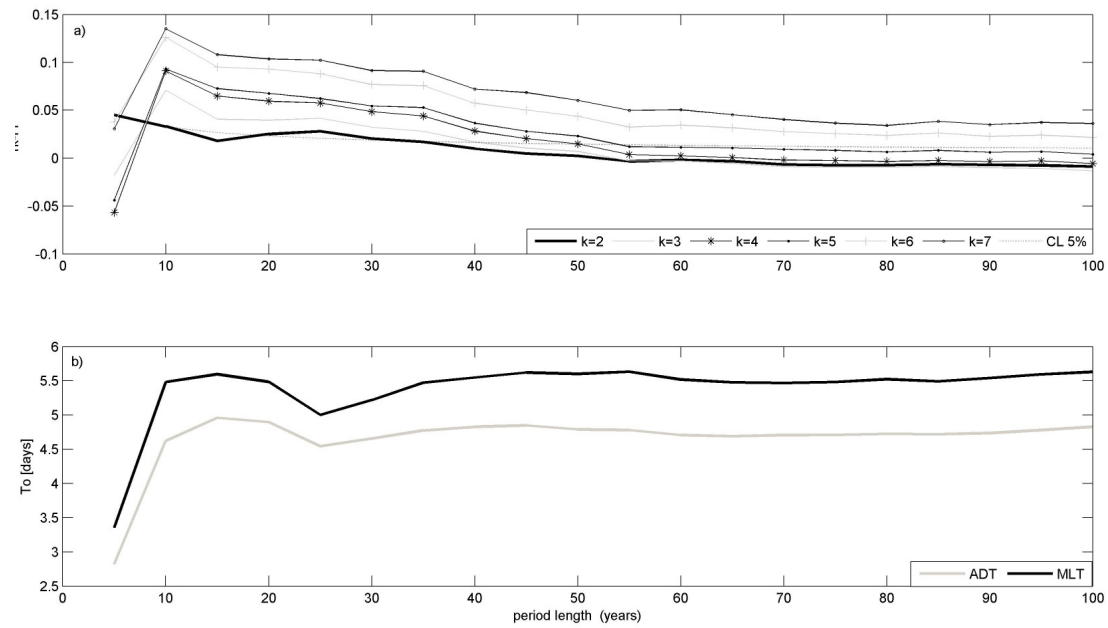

Figura 8 - (a) Diferencias entre el coeficiente de autocorrelación (rk) calculado y el asociado a un proceso Markoviano (r1k) para estimaciones con períodos de distinta longitud y (b) Estimación del tiempo de independencia (To) en función de la longitud de períodos para la temperatura mínima en S. M. de Tucumán para considerando anomalías aditivas (gris) y multiplicativas (negro).
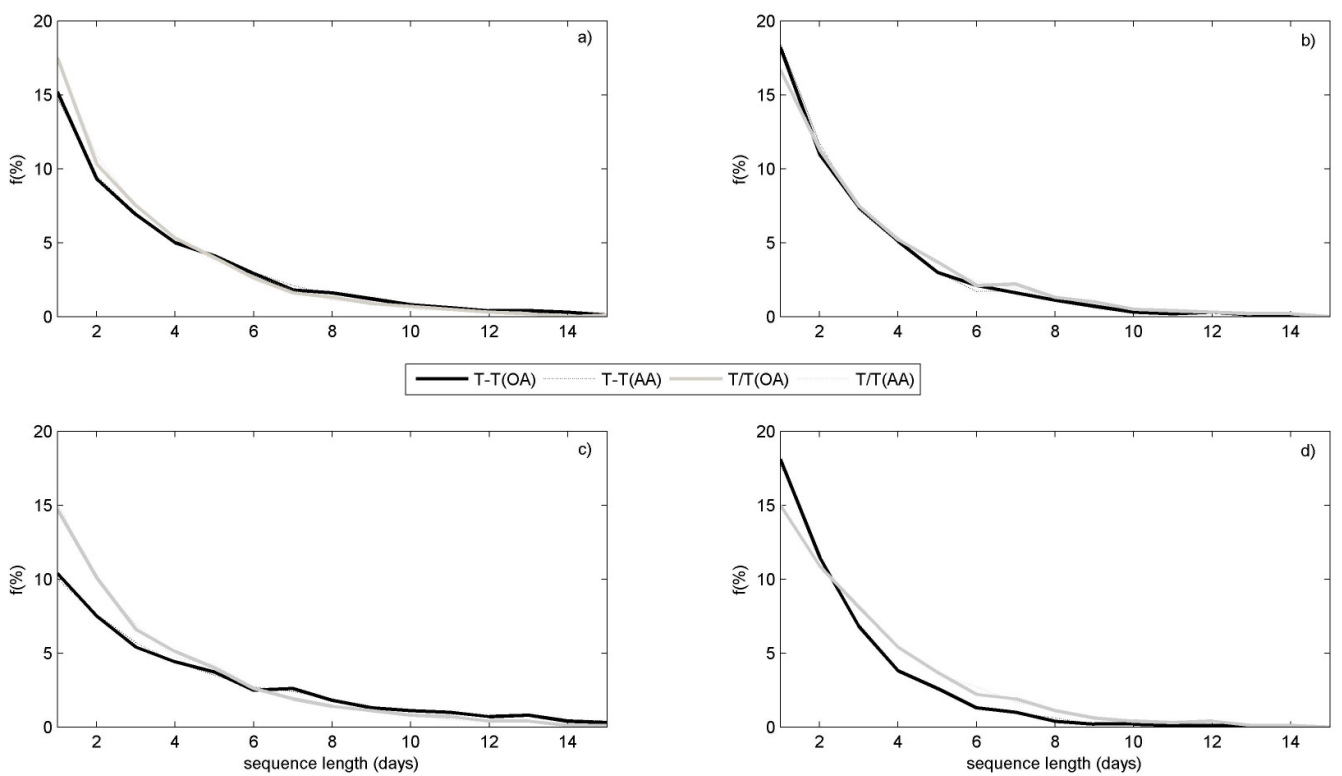

Figura 9 - Distribución de secuencias de anomalías positivas (a) y negativas (b) de temperatura máxima y de anomalías positivas (c) y negativas (d) de temperatura mínima.

Pero, ¿que significa que un año tenga más influencia de los efectos lineales/no lineales? En la Figura 9 se muestran las distribuciones de secuencias de anomalías positivas y negativas para las temperaturas máximas y mínimas, calculadas según el modelo lineal y aditivo. En general para tanto para Tx y Tn se observa una mayor persistencia de las anomalías cálidas si se considera el método multiplicativo. Esto esta asociado a procesos advectivos lentos donde las masas de aire cálido permanecen una gran cantidad de días, efecto que se evidencia mejor sobre la temperatura mínima. En cuanto a las secuencias de días fríos, no se observan grandes diferencias entre ambas estimaciones, aunque las variaciones lineales muestran mayor persistencia.

\section{CONCLUSIONES}

Para lograr un diagnóstico climático objetivo es necesario obtener un filtro o función de transferencia que introduzca la menor cantidad de ruido debido a que un análisis en escala diaria puede ser obscurecido por su elección. Para esto, se analiza la robustez y estabilidad de diferentes métodos para estimar la onda anual. Aquí se muestra la presencia de asimetrías en la onda anual de temperatura que en algunos casos son producto de interacciones no lineales entre la onda anual y las perturbaciones. Además se muestra que el cálculo de la onda anual a partir de la mediana produce una estimación más 
robusta para períodos de información menores a 50 años. Sin embargo, para períodos mayores de información no se observan diferencias entre los distintos estimadores.

Por otro lado, existe una dependencia entre el tiempo de independencia $\left(T_{o}\right)$ y el período analizado, es decir existe una variación interanual de $T_{o}$ que puede variar en la región entre 3 y 7 días. Este efecto posiblemente está asociado a variaciones entre períodos húmedo/secos. Además se observa un mayor $T_{o}$ si se considera el término no lineal de las anomalías, es decir el análisis de ésta componente conduce a estimar efectos más persistentes que se ven afectados por la pendiente de la onda anual.

En general los procesos asociados a una relación no lineal con la onda anual evidencian una mayor persistencia de las anomalías cálidas, principalmente sobre las temperaturas mínimas. Esto esta posiblemente asociado a procesos advectivos lentos donde las masas de aire cálido permanecen durante varios días sin transformación.

\section{AGRADECIMIENTOS:}

Al Proyecto UBA 20020100100628 y al Proyecto CONICET PIP 112-200801-00762, por la financiación de este trabajo.

\section{REFERENCIAS}

BAYLEY, G.; HAMMERSLEY J. The effective number of independent observations in an autocorrelated time series. Journal of the Royal Society, Ser. 1B, v. 8, p. 184-197, 1946.

BOX, G.; JENKINS, G. Time Series Analysis: Forecasting and Control. Holden-Day, 1970. 592p.

BROOKS C., N.; CARRUTHERS, N. Handbook of Statistical Methods in Meteorology. Her Majesty's Stationery Office, 1953. 412p.

CHATFIELD, C. The analysis of time series: An introduction. Chapman \& Hall, 1989. 304 pp.

HARVEY, A. Forecasting, structural time series models, and the Kalman filter. Cambridge University Press, 1990.572 p.

HOEL P. Introduction to mathematical statistics. Wiley New York, 1984. $448 \mathrm{p}$.

JENKINS, G.; WATTS, D. Spectral analysis and its applications. Holden-Day, 1968. 525 p.

KÖPPEN, W. Climatología. Fondo de Cultura Económica, México, 1948. $477 \mathrm{p}$.

LEITH C. E. The standard error of time-average estimates of climatic means. Journal of Applied Meteorology, v.12, p. 1066-1069, 1973.

LEITH, C. E. Objective methods for weather prediction. Annual Review of Fluid Mechanics, v. 10, p. 107-128, 1978.
MAKRIDAKIS, S.; WHEELWRIGHT, S. C. Forecasting Methods for Management $\left(\mathbf{5}^{\text {th }}\right.$ ed). New York: John Wiley \& Sons, 1989. $470 \mathrm{p}$.

MAKRIDAKIS, S.; WHEELWRIGHT, S.; MCGEE, V. Forecasting: Methods and Applications. John Wiley \& Sons, 1998. $642 \mathrm{p}$.

MANN, M.; PARK, J. Greenhouse warming and changes in the seasonal cycle of temperature: Model versus observations. Geophysical Research Letters, v. 23, p. 1111-1114, 1996.

MILLER D.; WILLIAMS, D. Shrinkage estimators of time series seasonal factors and their effect on forecasting accuracy. International Journal of Forecasting, v. 19, p. 669-684, 2003.

MINETTI, J.L. Estudio de las singularidades climáticas en series de temperaturas del Noroeste Argentino. 1991. 117 p. Tesis de doctorado en Meteorologia. FCEyN-UBA, 1991.

MINETTI, J. L.; VARGAS, W. M. Interaction Processes between the Annual Wave and the Disturbances in Series of Daily Temperature. International Journal of Climatology, v. 10, p. 299-305, 1997.

NAUMANN G.; VARGAS W.M.; MINETTI J. Persistence and long-term memories of daily maximum and minimum temperatures in southern South America. Theoretical and Applied Climatology v. 105, p. 341-355. doi: 10.1007/ s00704-010-0395-3, 2011.

RUSSELL J.S. Mathematical representation of related periodic variables using Fourier series and projections of vectors functions: application to the biological sciences. Agricultural and Forest Meteorology, v. 30, p. 215-224, 1984

SELLERS, W.K. Physical Climatology. University of Chicago Press, 1965. $272 \mathrm{p}$.

SPARKS, T.; MENZEL G. A. Observed changes in seasons: an overview. International Journal of Climatology, v. 22, p. 1715-1725, 2002.

STRAUS, D. M. On the role of the seasonal cycle. Journal of the Atmospheric Sciences, v. 40, p. 303-313, 1983.

STINE, A. R.; HUYBERS P.; FUNG Y. Changes in the phase of the annual cycle of surface temperature. Nature, v. 457, p. 435-440, 2009.

THOMSON, D. The seasons, global temperature, and precession. Science, v. 268, p. 59-68, 1995.

TRENBERTH, K.E. What are the seasons? Bulletin American Meteorological Society, v. 64, p. 1276-1282, 1983.

TRENBERTH, K. Some effects of finite sample size and persistence on meteorological statistics. Part I: Autocorrelations. Monthly Weather Review, v. 112, p. 2359-2368, 1984a.

TRENBERTH, K. Some effects of finite sample size and persistence on meteorological statistics. Part I: Potential 
predictability. Monthly Weather Review, v. 112, p. 23692379, 1984b.

VARGAS W. M.; NAUMANN G.; MINETTI J. Las Tendencias de la temperatura máxima y mínima en Series de Referencia del Sudeste de Sudamérica. Aspectos metodológicos. Revista de Geografía, v. 8, p. 67-74, 2006.

VARGAS W. M.; NAUMANN G. Impacts of climatic change and low frequency variability in reference series on daily maximum and minimum temperature in southern south America. Regional Environmental Change, v. 8, p. 4587, 2008.

WEI, W.W.S. Time Series Analysis: Univariate and Multivariate Methods. Addison-Wesley, 1993. 624 p. 